\title{
Clinical and histological features of nonalcoholic steatohepatitis in
} Iranian patients

\author{
Hossein Bahrami*, Nasser Ebrahimi Daryani, Shahram Mirmomen, \\ Farin Kamangar, Babak Haghpanah and Mehdi Djalili
}

Address: Digestive Diseases Research Center - Imam Hospital, Tehran University of Medical Sciences, Iran

Email: Hossein Bahrami* - dr_Bahrami@hotmail.com; Nasser Ebrahimi Daryani - nebrahim@sina.tums.ac.ir; Shahram Mirmomen - mirmomen@ams.ir; Farin Kamangar - farinkamangar@yahoo.com; Babak Haghpanah - babakhp@myrealbox.com; Mehdi Djalili - mehdidjalili@yahoo.com

* Corresponding author

Published: 16 October 2003

BMC Gastroenterology 2003, 3:27
Received: 14 March 2003

Accepted: 16 October 2003

This article is available from: http://www.biomedcentral.com/I47I-230X/3/27

(C) 2003 Bahrami et al; licensee BioMed Central Ltd. This is an Open Access article: verbatim copying and redistribution of this article are permitted in all media for any purpose, provided this notice is preserved along with the article's original URL.

\begin{abstract}
Background: Although several studies have been performed on risk factors and natural course of $\mathrm{NASH}$, it seems that NASH tends to be more than a disease confined to strict boundaries. The objective of this study was to assess the clinical and paraclinical features and risk factors for nonalcoholic steatohepatitis (NASH) patients in an Iranian population

Methods: Patients with histologically confirmed NASH who had elevated liver aminotransaminases, negative serologic markers of viral or autoimmune hepatitis and no findings in favor of metabolic liver disease were enrolled. A careful history was taken regarding alcohol intake.

Results: 53 patients consisting of 32 male and 2 I female entered the study. The mean age was 37.8 \pm II.3 years. Twenty-six patients (55.3\%) were overweight, I5 (3I.9\%) obese, 40 (75.5\%) dyslipidemic, and three patients (5.7\%) were diabetic. Liver biopsy showed mild steatosis in $35.7 \%$, moderate steatosis in $53.6 \%$, and severe forms in $10.7 \%$. In $80.2 \%$ of patients, portal inflammation was present, and $9.4 \%$ had cirrhosis. The amount of increase in liver enzymes bore no relationship with fibrosis, portal inflammation, and degree of steatosis.
\end{abstract}

Conclusions: The patients in our study showed a male predominancy and were somewhat younger than other studies.

\section{Background}

Non-alcoholic steatohepatitis (NASH) is a disease of unknown origin characterized histologically by changes similar to what is seen in alcoholic-like liver injury but in the absence of significant alcohol intake $[1,2]$. NASH is considered a type of chronic hepatitis and is a severe form of a spectrum called non-alcoholic fatty liver disease
(NAFLD) [3]. NAFLD has four histological stages: (1) fatty infiltration of the liver (2) fatty infiltration plus inflammation (3) fatty infiltration with ballooning degeneration (4) fatty infiltration with lesions similar to alcoholic hepatitis and sinusoidal fibrosis, polymorphonuclear infiltration with or without Mallory hyaline. NASH is the name given to the third and fourth stages $[4,5]$. 
The prevalence and clinical significance of NASH appears to be more than what is generally believed [6-11]. Among patients who have had liver biopsies, NASH is seen in approximately 7 to 9 percent in Western countries $[12,13]$. Ultrasonographic studies on normal population show a prevalence of 25 percent for NAFLD in the United States $[5,14]$. The disease predominantly occurs between the ages of 40 and $60,[9,11,15]$ although there have been reports in children over the age of $10[16,17] \mathrm{NASH}$ is frequently associated with disorders such as insulin resistance, obesity, type 2 diabetes mellitus, hyperlipidemia, protein-calorie malnutrition, and jejeunoileal bypass surgery $[5,9,12,18-21]$ It is important to consider that NASH can progress to cirrhosis and hepatic failure $[7,8]$, further underscoring its importance as a potentially serious and life-threatening disease.

Regarding this fact that in Iran alcohol consumption is much less than western countries, we doubted whether the demography and risk factors of NASH in Iranian populations is the same as what has been seen in previous studies.

\section{Methods \\ Patient selection}

The cohort of patients reviewed here were selected from all patients between 15 and 65, referred to Gastroenterology and Hepatology Clinic of Imam Khomeini Hospital, Tehran, Iran, from March 20, 1999 to November 21, 2001. Inclusion criteria were: 1 . elevated liver aminotransaminases 2 . histologic confirmation of nonalcoholic steatohepatitis 3. negative serologic markers of viral or autoimmune hepatitis (including $\mathrm{HBsAg}$, HCV antibody (ELISA), HIV antibody (ELISA), antinuclear antibodies, anti-smooth muscle antibodies, and anti-liver/ kidney microsomes type 1 antibodies) 4 . no findings in favor of metabolic liver diseases, including Wilson's disease and hemochromatosis) and negative alpha- 1 antitrypsin. A careful history was taken with special attention to alcohol intake and ultrasonography was performed in all enrolled patients. Alcohol intake was asked from the patient and at least one of the patients' companions. In case of positive alcohol consumption, the questionnaire used for this purpose had more detail to help the interviewer to estimate the approximate amount of alcohol consumption. Exclusion criteria were: 1. recent gastrointestinal surgery 2. pregnancy 3 . usage of drugs known to result in steatosis, including glucocoticoids, synthetic esterogens, aspirin, tamoxifen, amiodarone, Calciumchannel blockers, and methotrexate.

Of 213 patients (108 male and 105 female) who have primarily been studied for elevation of serum aminotransferases in the beginning, 72 patients who had inclusion criteria, except pathologic confirmation, consented to undergo liver biopsy. The pathologic diagnosis of NASH was confirmed in 53 patients and these patients were analyzed. Also we used the demographic data of the other 21 patients to avoid any selection bias in this characteristics, which sounded to be different in our study, comparing with previous studies.

\section{Laboratory and pathological studies}

Levels of aminotransferases, alkaline phosphatase, total bilirubin, serum cholesterol and triglycerides, fasting blood sugar, and prothrombin time were measured using standard techniques. The definite diagnosis of NASH was based on a histology showing moderate to gross macrovesicular fatty change with inflammation (lobular or portal), with or without Mallory bodies, fibrosis, or cirrhosis. The grading and staging of all biopsy specimens were determined based on the method proposed by Brunt et al. [2] All patients who had mild steatosis in their biopsy were re-checked for HCV infection using radioimmunoabsorbant assay (RIBA), which was negative in all. Because of financial limitation we did not recheck those with higher degrees of steatosis, since the diagnosis of NASH was more probable in them. A single radiologist performed all ultrasonographic studies and all pathologic studies were performed and reported by one pathologist. Overweight was defined as a BMI between 25 and $29.9 \mathrm{~kg} /$ $\mathrm{m}^{2}$, and obesity as BMI equal or above $30 \mathrm{~kg} / \mathrm{m}^{2}$. Ideal weight for height was calculated by Hamwi Method [22] (Men: $48.18 \mathrm{~kg}$ for $150 \mathrm{~cm}+/-1.1 \mathrm{~kg}$ per $\mathrm{cm}$ over/under $150 \mathrm{~cm}$; Women: $45.45 \mathrm{~kg}$ for $150 \mathrm{~cm}+/-0.91 \mathrm{~kg}$ per $\mathrm{cm}$ over/under $150 \mathrm{~cm}$ ). Diabetes mellitus was defined as either 1)fasting blood glucose concentration of $126 \mathrm{mg} /$ $\mathrm{dL}$ or higher in two occasions, or 2)a random value of 200 $\mathrm{mg} / \mathrm{dL}$ or higher, confirmed on another occasion, or 3) having a confirmed history of diabetes mellitus for which the patient is currently receiving antiglycemic drugs. Dyslipidemia and insulin resistance was defined according to guidelines of ATPIII.[23]> Thus, patients with one of the criteria of LDL-C $\geq 160 \mathrm{mg} / \mathrm{dL}$, total cholesterol $\geq 200 \mathrm{mg} /$ $\mathrm{dL}$, triglycerides $\geq 150 \mathrm{mg} / \mathrm{dL}$, or HDL-C $<40 \mathrm{mg} / \mathrm{dL}$ were considered dyslipidemic. Insulin resistance was defined with presence of three of the following:

1. Abdominal obesity, defined as a waist circumference in men $>102 \mathrm{~cm}$ (40 in) and in women $>88 \mathrm{~cm}$ (35 in)

2. Triglycerides $\geq 150 \mathrm{mg} / \mathrm{dL}$

3. HDL cholesterol $<40 \mathrm{mg} / \mathrm{dL}$ in men and $<50 \mathrm{mg} / \mathrm{dL}$ in women

4. Blood pressure $\geq 130 / \geq 85 \mathrm{mmHg}$

5. Fasting glucose $\geq 110 \mathrm{mg} / \mathrm{dL}$ 
Table I: Anthropometric and biochemical characteristics of 53 patients with NASH

\begin{tabular}{llll}
\hline & Male & Female & Total \\
\hline Number & 32 & 21 & 53 \\
Age (y) & $36.0 \pm 10.6$ & $40.5 \pm 11.9$ & $37.8 \pm 11.3$ \\
Weight (kg) & $93.6 \pm 16.5^{*}$ & $69.3 \pm 12.0$ & $83.8 \pm 19$ \\
BMI (kg/m²) & $30.7 \pm 4.9 \dagger$ & $27.3 \pm 3.5$ & $29.3 \pm 4.7$ \\
Normal BMI, $\mathrm{n}(\%)$ & $7(21.9 \%)$ & $5(23.8 \%)$ & $12(22.6 \%)$ \\
Obesity, $\mathrm{n}(\%)$ & $13(46.4)^{*}$ & $2(10.5)$ & $15(31.9)$ \\
Overweight, $\mathrm{n}(\%)$ & $12(42.9)^{*}$ & $14(73.7)$ & $26(55.3)$ \\
Total cholesterol (mg/dL) & $229.6 \pm 73.5$ & $223.5 \pm 62.5$ & $227.0 \pm 68.5$ \\
LDL-C (mg/dL) & $139.5 \pm 42.3$ & $123.0 \pm 43.3$ & $134.8 \pm 42.2$ \\
HDL-C (mg/dL) & $47.6 \pm 16.1$ & $43.1 \pm 13.6$ & $46.3 \pm 15.2$ \\
Triglycerides (mg/dL) & $207 \pm 83.6$ & $184 \pm 89.1$ & $197.3 \pm 85.8$ \\
FBS (mg/dL) & $102.1 \pm 11.4$ & $112.8 \pm 42.5$ & $106.5 \pm 28.1$ \\
ALT (IU/L) & $80.3 \pm 27.5$ & $82.0 \pm 38.8$ & $80.9 \pm 32.0$ \\
AST (IU/L) & $68.8 \pm 22.4$ & $71.3 \pm 37.5$ & $69.8 \pm 28.9$ \\
AST/ALT & $0.9 \pm 0.3$ & $0.9 \pm 0.5$ & $0.9 \pm 0.4$ \\
\hline
\end{tabular}

$*_{p}<0.001, \dagger p<0.05$ compared to female patients. ALT: Alanine Aminotransferase, AST: Aspartate Aminotransferase, BMI: Body Mass Index, FBS: Fasting Blood Sugar, HDL-C: High Density Lipoprotein Cholestrol, LDL-C: Low Density Lipoprotein Cholestrol.

Risk factors for NASH, according to previous studies, included diabetes mellitus, hyperlipidemia, obesity, history of significant weight loss or weight gain, history of consumption of esterogens or androgens, and extensive abdominal surgery.

\section{Statistical analysis}

The results of quantitative variables are presented as mean \pm SD and those of qualitative variables as numbers and percentages. T test was used to compare quantitative variables between subgroups of patients, and differences between categorical variables were analyzed by Chi Square. Logistic regression analysis was used to assess the effect of different factors on histological findings in liver biopsy specimens. A significance level of 0.05 was used.

\section{Results}

Of the 53 patients whose diagnosis was confirmed by biopsy, 32 (60.4\%) were male and 21 (39.6\%) were female with the mean age of $37.8 \pm 11.3$ years. Mean weight of the patients was $83.8 \pm 19 \mathrm{~kg}$ and mean BMI was $29.3 \pm 4.7 \mathrm{~kg} / \mathrm{m}^{2}$. Twenty-six patients $(55.3 \%)$ were overweight and $15(31.9 \%)$ were obese (Table 1$)$. None of the patients had weights lower than the "ideal weight for height". On the average, the patients had weights $36.3 \pm$ $20.5 \%$ (ranging from 0 to $125.2 \%$ ) higher than the ideal weight and $94.3 \%$ of the patients had weights more than $10 \%$ higher than the ideal weight for their height (the mean ideal weight for height of the patients was $61.4 \pm 9.6$ $\mathrm{kg})$. Six patients $(11.3 \%)$ had a history of considerable weight loss and 12 patients $(22.7 \%)$ reported an increase in weight during the last year.
Three patients $(5.7 \%)$ had overt diabetes mellitus. Forty patients $(75.5 \%)$ had some form of dyslipidemia; in $66.0 \%$ serum cholesterol was high, in $60.4 \%$ hypertriglyceridemia was present, and both cholesterol and triglycerides were high in 50.9\%. Insulin resistance was observed in 29 patients $(54.7 \%)$. Seven patients $(13.2 \%)$ had none of the risk factors usually associated with NASH. The mean ALT and AST levels were $80.9 \pm 32.0 \mathrm{IU} / \mathrm{L}$ and 69.8 $\pm 28.9 \mathrm{IU} / \mathrm{L}$, respectively. Mean AST to ALT ratio was $0.9 \pm$ 0.4 . This ratio was below 1 in $65.3 \%$ of patients and below 2 in $96.2 \%$.

In 31 patients (58.5\%) abnormal ultrasound findings were observed; $17 \%$ of all patients had increased liver size, and 50.9\% had hyperechogenicity, indicating fatty liver.

$79.2 \%$ of patients had no or very unspecific clinical manifestations, like fatigue, malaise and weakness. Different clinical manifestations are shown in Figure 1.

Liver biopsy showed that steatosis was mild in $35.7 \%$, moderate in $53.6 \%$, and severe in $10.7 \%$. 22 patients (41.5\%) had mild, 26 patients $(49.1 \%)$ moderate and 5 patients $(9.4 \%)$ had severe necroinflammatory grades. In histological staging of fibrosis, $11.3 \%, 18.9 \%, 22.6 \%$, and $37.7 \%$ of patients had 0 through 3 scores, respectively and in $9.4 \%$ (5 patients), liver biopsy demonstrated cirrhosis (stage 4). The results of logistic regression analysis showed no relationship between the level of liver enzymes and fibrosis, necroinflammatory grading, and steatosis. Presence of insulin resistance did not have any significant relationship with the staging and grading of the disease, as well. 


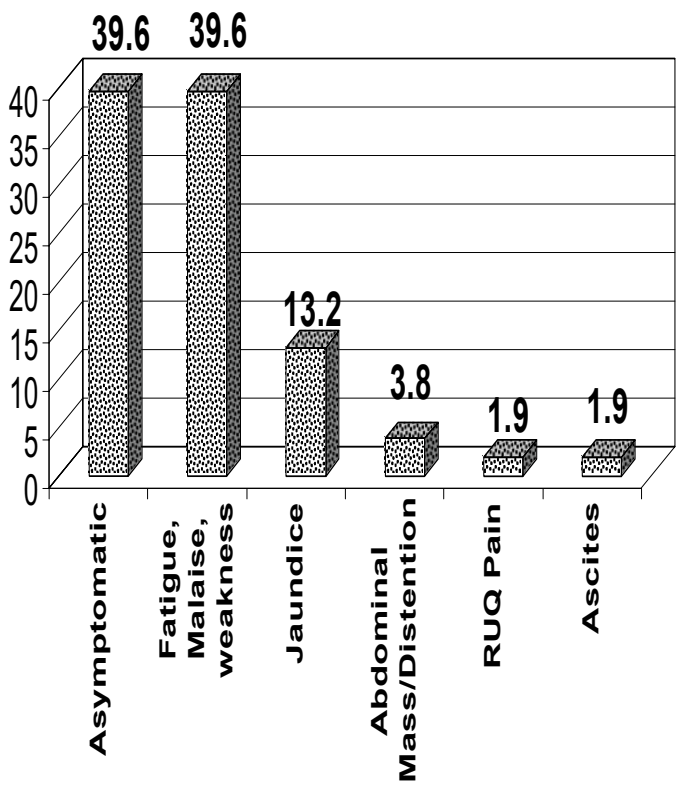

\section{Figure I}

Relative Frequency (Percent) of Different Clinical Manifestations in Patients with NASH, Tehran, 2002

In an analysis of demographic characteristics of 53 histologically-confirmed NASH cases and 21 patients with clinical diagnosis of NASH whose diagnosis was not confirmed in biopsy, $56.8 \%$ of patients $(42 / 74)$ were male and $43.2 \%$ (32/74) were female. Mean age was 39.2 \pm 12.1 years and mean BMI was $29.1 \pm 4.1 \mathrm{~kg} / \mathrm{m}^{2}$. There was no significant difference in these characteristics between those who had positive biopsy and those who had negative.

\section{Discussion}

Nonalcoholic fatty liver disease (NAFLD) seems to have become an important medical entity and this importance is mainly resulted from its potential to progress to cirrhosis and liver failure and its common occurrence in general population [6]. Because of the low rate of alcohol consumption in Iran, the prevalence might be even higher than other countries. Regardless of the religious beliefs, the habit of alcohol consumption in Iranian population, in most instances, is not so heavy and frequent as what is seen in most of the western countries.

Our patients were somewhat younger than those in previous studies (40-60 years old) $[9,12,15]$. Also most of our patients were male $(60.4 \%)$, while most of the previous series showed a female predominance $[10-12,19]$ and only a few had more male patients than females [9]. One of the reasons for these differences might be the failure to rigorously rule out hepatitis $\mathrm{C}$ in some of the studies. We rechecked all patients with mild steatosis for hepatitis $\mathrm{C}$ infection. In the report mentioned before as showing a male preponderance [9], also hepatitis $\mathrm{C}$ was excluded with precision, and the patients had demographic characteristics different from those usually reported. The male predominancy in our study, prompted us to evaluate whether this difference is related to the disease, itself, or is a selection bias in patients who are referred to our clinic. We compared these patients with other patients who primarily had elevation of serum aminotransferases but did not meet the inclusion criteria of the study. From total number of 127 patients with aminotransferases elevation who were excluded from the study, $52.8 \%$ were male. Although not exclusively rejecting the selection bias, these findings are against the assumption of a selection bias, since there is not a significant male predominance in other patients of our clinic. But more investigations are mandatory in this regard. Another probable justification of the different sex composition of our patients might be attributed to the lower rate of alcohol consumption in our society. Since men usually consume more amount of alcohol and are more at risk of alcoholic liver disease, in societies with higher prevalence of alcohol consumption, more men are excluded from the diagnosis of nonalcoholic steatohepatitis because of their alcohol consumption.

The mean weight of the patients was higher than normal as in previous reports. $[11,12,18]$ Most patients had abnormally high BMI's and $94.2 \%$ had weights more than $10 \%$ above their ideal body weights. $11.3 \%$ of the patients had a history of weight loss in the year before diagnosis, which is similar to other results $[24,25]$.

Another difference between our results and other reports is the relatively low prevalence of diabetes. Although some reports have shown a prevalence of $2-5 \%$ for diabetes mellitus in this group $[19,26]$, in most of them this prevalence is as high as $21-55 \%[12,9-11,18-21]$. An interesting finding is that $13.2 \%$ of our patients had none of the known risk factors for development of NASH, and NASH should be considered as an entity not being confined to obese and diabetic patients.

Liver enzyme levels in our patients were similar to previous reports [9]. The mean AST to ALT ratio was less than 1 as expected, and in $96.2 \%$ this ratio was below 2 . In alcoholic liver disease, this ratio is usually above two, averaging from 2.6 to $2.85[24,27,28]$.

We observed dyslipidemia in $75.5 \%$ of our patients. Others have reported similar prevalences $(20-80 \%)$ [9$12,20,21]$. The ultrasonographic findings, such as fatty infiltration, are usually nonspecific [29]. 
It is not usually advisable to make a diagnosis of NASH before liver biopsy. The possibility of making an erroneous diagnosis is higher in NASH as compared to alcoholic liver disease. In our study, from 74 patients selected on clinical basis, 53 ones had NASH on liver biopsy. This figure is larger than the ones mentioned in other studies [30-33], which is probably due to precise patient selection in the present study, exclusion of cases with low aminotransaminase levels, or positive markers for viral hepatitis.

Factors affecting the pathogenesis of NASH are steatosis, inflammation, and fibrosis [2,34-38]. The severity of steatosis was similar to other reports. Also, the majority of patients had portal inflammation. The findings on liver biopsy were not correlated to liver enzymes, thus the increase in liver enzymes does not seem a good estimate of the severity of liver disease.

NASH can cause fibrosis and progress to cirrhosis. It has been shown that this progress is seen in $8-26 \%$ of patients $[6,8,9,19]$. Hepatic fibrosis, Mallory hyaline, or ballooning degeneration are findings that indicate higher likelihood of progression to cirrhosis [39]. Altogether, these findings further underscore the importance of NASH and the need to diagnose it before it causes irreversible liver damage.

\section{Conclusion}

The epidemiology and risk factors of nonalcoholic steatohepatitis might be different in societies with lower rate of alcohol consumption, although this is just a preliminary hypothesis that should be evaluated in further studies. Regarding the considerable prevalence of the disease, confinement of the diagnosis to some specific epidemiologic and demographic subgroups does not seem so reasonable, particularly in regions where the distribution of the disease is not well-known.

\section{Competing Interests}

None declared.

\section{Acknowledgement}

The authors appreciate the indefatigable efforts and adroit help of Arash Etemadi, MD, and Alireza Moayyeri, MD, in this study.

\section{References}

I. Sheth SG, Gordon FD and Chopra S: Nanalcoholic steatohepatitis. Ann Intern Med 1997, I 26: I37-45.

2. Brunt EM, Janney CG and Di Bisceglie AM et al.: Nonalcoholic steatohepatitis:a proposal for grading and staging the histological lesions. Am J Gastroenterol I 999, 94:2467-74.

3. Clark JM, Brancati FL and Diehl AM: Nonalcoholic fatty liver disease. Gastroenterology 2002, I 22: I649-57.

4. Flack-ytter $\mathrm{Y}, \mathrm{McC}$ Cullough AJ and Younossi Z et al:: Clinical features and natural history of Nonalcoholic Steatosis Syndromes. Sem Liver Disease 200I, 2 I : I7-26.

5. Kumar KS and Malet PF: Nonalcoholic steatohepatitis. Mayo Clin Proc 2000, 75:733-39.
6. Angulo P: Nanalcoholic fatty liver disease. N Engl ] Med 2002, 346:|22|-3|.

7. Reid AE: Nonalcoholic steatohepatitis. Gastroenterology 200I, I 2 I:7| 0-723.

8. Matteoni C, Younossi ZM and McCullough A: Nonalcoholic fatty liver disease:A spectrum of clinical pathological severity. Gastroenterology 1999, I 16:1413-19.

9. Bacon B, Faravash MJ and Janney CG et al:: Nonalcoholic steatohepatitis: An expanded clinical entity. Gastroenterology 1994, 107: I 103-6.

10. Powell EE, Cooksley GE and Hanson R et al:: The natural history of nonalcoholic steatohepatitis: A follow-up study of $\mathbf{4 2}$ patients for up to 21 years. Hepatology 1990, I I:74-80.

II. Lee RG: Nonalcoholic steatohepatitis: A study of $\mathbf{4 9}$ patients. Hum Pathol 1989, I I:594-8.

12. Ludwig J, Viggiono TR and McGill DB et al.: Nonalcoholic steatohepatitis: Mayo Clinic experiences with a hitherto unnamed disease. Mayo Clin Proc 1980, 55:342-8.

13. Propst $A$, Propst $T$ and Judmaei $G$ et al.: Prognosis in nonalcoholic steatohepatitis (letter). Gastroenterology 1995, 108:1607.

14. el-Hassan AY, Ibrahim EM and al-Milhim FA et al.: Fatty infiltration of the liver: analysis of prevalence, radiological and clinical features and influence on patient management. $\mathrm{Br} J$ Radiol 1992, 65:774-8.

15. Nonomura A, Mizukami $Y$ and Unoura $M$ et al.: Clinicopathologic study of alcohol-like liver disease in non-alcoholics; Nonalcoholic steatohepatitis and fibrosis. Gastroenterol Jpn 1992, 27:521.

16. Baldrige AD, Perez-Atayde AR and Graeme-Cook F et al.: Idiopathic steatohepatitis in childhood: $A$ multicenter retrospective study. J Pediatr 1995, I 27:700.

17. Moran JR, Ghishan FK and Halter SA et al.: Steatohepatitis in obese children: $\mathbf{A}$ cause of chronic liver dysfunction. $\mathrm{Am} J$ Gastroenterol 1983, 78:374.

18. Wanless IR and Lentz JS: Fatty liver hepatitis (Steatohepatitis) and obesity: An autopsy study with analysis of risk factors. Hepatology 1990, I2: I I06.

19. Itoh S, Yougel T and Kawagoe K: Comparison between nonalcoholic steatohepatitis and alcoholic hepatitis. Am J Gastroenterol 1987, 82:650.

20. Diehl AM, Goodman Z and Ishak KG: Alcohol-like disease in nonalcoholics. Gastroenterology 1988, 95: 1056.

21. Pinto HC, Baptista A and Camilo ME et al.: Nonalcoholic steatohepatitis. Clinicopathological comparison with alcoholic hepatitis in ambulatory and hospitalized patients. Dig Dis Sci 1996, $41: 172$.

22. Hamwi G]: Therapy: Changing dietary needs. In: Diabetes Mellitus: Diagnosis and Treatment Edited by: Danowski TS. New York, NY, American Diabetes Association; 1964:73-78.

23. Executive Summary of The Third Report of The National Cholesterol Education Program (NCEP) Expert Panel on Detection, Evaluation, And Treatment of High Blood Cholesterol In Adults (Adult Treatment Panel III). JAMA 200I, 285:2486-97.

24. Cello JP and Grendell JH: The liver in systemic conditions. In: Hepatology Edited by: Zakim D, Boyer TD. Philadelphia, WB Saunders; 1990:1428.

25. Capron JP, Delamarre $M$ and Dupas JL et al:: Fasting in obesity: another cause of liver injury with alcoholic hyaline? Dig Dis Sci 1982, 54:374-7.

26. Adler $M$ and Schaffner $F$ : Fatty liver hepatitis and cirrhosis in obese patients. Am J Med 1979, 67:8I I-9.

27. Sorbi $D$, Boynton J and Lindor KD: The ratio of aspartate aminotransferase to alanine aminotransferase: Potential value in differentiating nonalcoholic steatohepatitis from alcoholic liver disease. Am J Gastroenterol 1999, 94:1018.

28. Cohen JA and Kaplan MM: The SGOT/SGPT ratio - an indicator of alcoholic liver disease. Dig Dis Sci 1979, 24:835.

29. Lonardo A, Bellini M and Tondelli E et al.: Nonalcoholic steatohepatitis and the "bright liver syndrome": Should a recently expanded clinical entity be further expanded? $\mathrm{Am} J$ Gastroenterol 1995, 90:2072.

30. Van Ness MM and Diehl AM: Is liver biopsy useful in the evaluation of patients with chronically elevated liver enzymes? Ann Intern Med 1989, I I I:473. 
31. James OF and Day CP: Nonalcoholic steatohepatitis (NASH): a disease of emerging identity and importance. J Hepatol 1998, 29:495-50।.

32. Neuschwander-Tetri BA and Bacon BR: Nonalcoholic steatohepatitis. Med Clin North Am 1996, 80: I I47-66.

33. Nomura F, Ohnishi $K$ and Ochiai T et al.: Obesity-related Nonalcoholic fatty liver:CT features and follow-up studies after low-calorie diet. Radiology 1987, 162:845-7.

34. Acosta $D$ and Wenzel DG: Injury produced by free fatty acids to lysosomes and mitochondria in cultured heart muscle and endothelial cells. Atherosclerosis 1974, 20:417.

35. Dianzani MU: Hepatotoxicology. In: Biochemical aspects of fatty liver Edited by: Meeks RG, Harrison SD, Bull RJ. Boca Raton, CRC; 1991:327.

36. Lombardi $B$ : Fatty liver: Considerations on the pathogenesis of fatty liver. Lab Invest 1966, I5: I.

37. Cortez-Pinto H, Chatham J and Chacko VP et al.: Alterations in liver ATP homeostasis in human nonalcoholic steatohepatitis: A pilot study. JAMA 1999, 282:1659.

38. Berson $A D, B e c o V$ and Lettetan $P$ et al.: Steatohepatitis-inducing drugs cause mitochondrial dysfunction of lipids peroxidation in rat hepatocytes. Gastroenterology 1998, I 1 4:764.

39. Falk-ytter Y, McCullough AJ and Younossi J et al.: Clinical features and natural history of nonalcoholic steatosis syndromes. Sem Liver Disease 200I, 21 : 17-26.

\section{Pre-publication history}

The pre-publication history for this paper can be accessed here:

http://www.biomedcentral.com/1471-230X/3/27/pre pub

Publish with Bio Med Central and every scientist can read your work free of charge

"BioMed Central will be the most significant development for disseminating the results of biomedical research in our lifetime. "

Sir Paul Nurse, Cancer Research UK

Your research papers will be:

- available free of charge to the entire biomedical community

- peer reviewed and published immediately upon acceptance

- cited in PubMed and archived on PubMed Central

-yours - you keep the copyright 